\title{
Prediction of disease-related genes based on weighted tissue-specific networks by using DNA methylation
}

\author{
Min Li ${ }^{1}$, Jiayi Zhang ${ }^{1}$, Qing Liu', Jianxin Wang ${ }^{1 *}$, Fang-Xiang Wu ${ }^{1,2^{*}}$ \\ From IEEE International Conference on Bioinformatics and Biomedicine (BIBM 2013) \\ Shanghai, China. 18-21 December 2013
}

\begin{abstract}
Background: Predicting disease-related genes is one of the most important tasks in bioinformatics and systems biology. With the advances in high-throughput techniques, a large number of protein-protein interactions are available, which make it possible to identify disease-related genes at the network level. However, network-based identification of disease-related genes is still a challenge as the considerable false-positives are still existed in the current available protein interaction networks (PIN).

Results: Considering the fact that the majority of genetic disorders tend to manifest only in a single or a few tissues, we constructed tissue-specific networks (TSN) by integrating PIN and tissue-specific data. We further weighed the constructed tissue-specific network (WTSN) by using DNA methylation as it plays an irreplaceable role in the development of complex diseases. A PageRank-based method was developed to identify disease-related genes from the constructed networks. To validate the effectiveness of the proposed method, we constructed PIN, weighted PIN (WPIN), TSN, WTSN for colon cancer and leukemia, respectively. The experimental results on colon cancer and leukemia show that the combination of tissue-specific data and DNA methylation can help to identify disease-related genes more accurately. Moreover, the PageRank-based method was effective to predict diseaserelated genes on the case studies of colon cancer and leukemia.
\end{abstract}

Conclusions: Tissue-specific data and DNA methylation are two important factors to the study of human diseases. The same method implemented on the WTSN can achieve better results compared to those being implemented on original PIN, WPIN, or TSN. The PageRank-based method outperforms degree centrality-based method for identifying disease-related genes from WTSN.

\section{Background}

With the completion of HGP (Human Genome Project) and the development of high-throughput technologies, more and more protein-protein interaction data can be obtained, which makes it possible for us to study the life activity at the network level [1-3]. Many network-based methods have been proposed to predict protein functions, identify essential proteins and disease-related genes and complexes [5-7]. It has been shown that the network-based

\footnotetext{
* Correspondence: jxwang@mail.csu.edu.cn; faw341@mail.usask.ca 'School of Information Science and Engineering, Central South University, Changsha 410083, Hunan, P. R. China

Full list of author information is available at the end of the article
}

disease-related gene discovery approaches can achieve comparable qualities with current integrative methods [8-11]. More and more attentions have been paid to discover disease-related genes by using network-based methods. Moti et al. [12] developed a neighbourhood-based algorithm to predict disease genes using protein-protein interactions by using the associated intervals. In a similar way, Kar et al. [13], Chavali et al. [14], and Sun et al. [15] further analyzed different diseases and demonstrated that the topological features of genes in associated intervals were different in the corresponding networks. Xu et al. [16] predicted disease-related genes by using topological features to improve KNN clustering algorithm. Sun et al. 
[17] used clustering analysis method to predict human disease-related gene clusters based on the network. In addition, some typical graph partitioning methods and clustering approaches, such as GS[18], MCL [19], VI-Cut [20], IPCA [21], MSCF [22], HC-PIN[23], RW[24], and their improved algorithms, can also be used to discover candidate disease-related genes.

Although great progresses have been made on the network-based methods, it is still a challenge task to identify disease-related genes as the considerable false-positives are still existed in the current available PINs [25]. To reduce the effect of false-positives, researchers started to integrate different types of biological information, such as gene expression profiles [26-29], orthology data [30], gene ontology annotations [31], and DNA methylation [32], into protein interaction networks. Accumulated studies suggested that DNA methylation may cause changes of chromatin structure, DNA conformation, DNA stability, and interaction mode between DNA and proteins, and such aberrant conditions may cause cancers[32]. Therefore, DNA methylation information can be used to improve identification of disease-related genes. For prioritizing cancer-related genes, Liu et al. [32] constructed a weighted human protein interaction network by using DNA methylation correlations.

Recently, some researchers tried to develop new network-based methods for finding disease-related genes by using tissue-specific networks (TSN). Tissue specificity is an important aspect of many genetic diseases and the majority of genetic disorders tend to manifest only in a single or a few tissues [33-35]. Magger et al. [36] believed that the predicted precision would be influenced when the same data sets were used to predict disease-related genes of different tissues and diseases. Tissue-specific networks can reflect the features of related tissues of diseases better, and usage of the network will enhance the accuracy of predicting disease-related genes.

In this paper, we constructed a weighted tissue-specific network (WTSN) by integrating human protein interaction network, DNA methylation, and tissue-specific data. A PageRank-based method was developed to identify disease-related genes from the constructed WTSN. To validate the effectiveness of the constructed network and the proposed method, we tested them on the prediction of disease-related genes of colon cancer and leukemia. The experimental results show that tissue-specific data and DNA methylation are two important factors to the study of human disease. The same method implemented on the WTSN can achieve better results compared to those being implemented on original PIN, WPIN, or TSN. The PageRank-based method outperforms degree centrality-based method for identifying disease-related genes from WTSN.

\section{Methods}

\section{Materials}

The human protein-protein interactions were downloaded from DIP [37], IntAct [38], MINT [39], BioGRID [40], HPRD [41], Uniprotkb and HGNC databases. These protein-protein interactions were combined to construct a PIN by filtering the self-interactions and repeated interactions. The final PIN comprises 15,389 human proteins and 108,317 physical interactions.

To determine tissue-specific interactions, gene expression microarray data GSE1133 and gene identity matched data GPL96 [42] were used to extract expression values of each gene in different tissues. All the co-expression relationships in 79 tissues were marked by binary variables. 79 tissues were mathematically represented in a matrix of 108,317 interactions.

DNA methylation information was downloaded from GSE17648 [43] and GSE28462 data sets of GEO. Aberrant methylation information was downloaded from PubMeth [44] database. Gene signing messages of related diseases in the gene signatures bank were downloaded from GeneSigDB [45].

\section{Construction of WTSN}

As shown in Figure 1, we constructed the WTSNs according to the following three steps:

Step 1: Construct the human PIN by integrating proteinprotein interaction data obtained from DIP [37], IntAct [38], MINT [39], BioGRID [40], HPRD [41], Uniprotkb and HGNC databases and filtering the self-interactions and repeated interactions.

Step 2: For a special disease, construct its TSN by using the PIN, tissue-specific data and disease-tissue association data.

Step 3: Weight the constructed TSN by using DNA methylation data.

In step 2, to construct the TSN for a special disease, we first obtained the relationship between diseases and tissues from the works of Lage et al [46]. From the disease-tissue association data we can extract the tissues which are related to the special disease. Tissue-specific subnetworks were constructed by using gene expression microarray data GSE1133 and GPL96. When constructing the tissuespecific subnetworks we also used the mode removal method which has been used by Waldman et al. [34], Bossi et al. [35] and Lopes et al. [47]. The main idea of the mode removal method is to construct tissue-specific subnetworks through removing unexpressed genes in related tissues. There are 79 different human cells or gene expression data of tissues. For each gene, its normalized expression level is calculated and it is considered to be expressed in the tissue [33] if its normalized expression level is larger than a certain threshold. For each tissue, a corresponding 


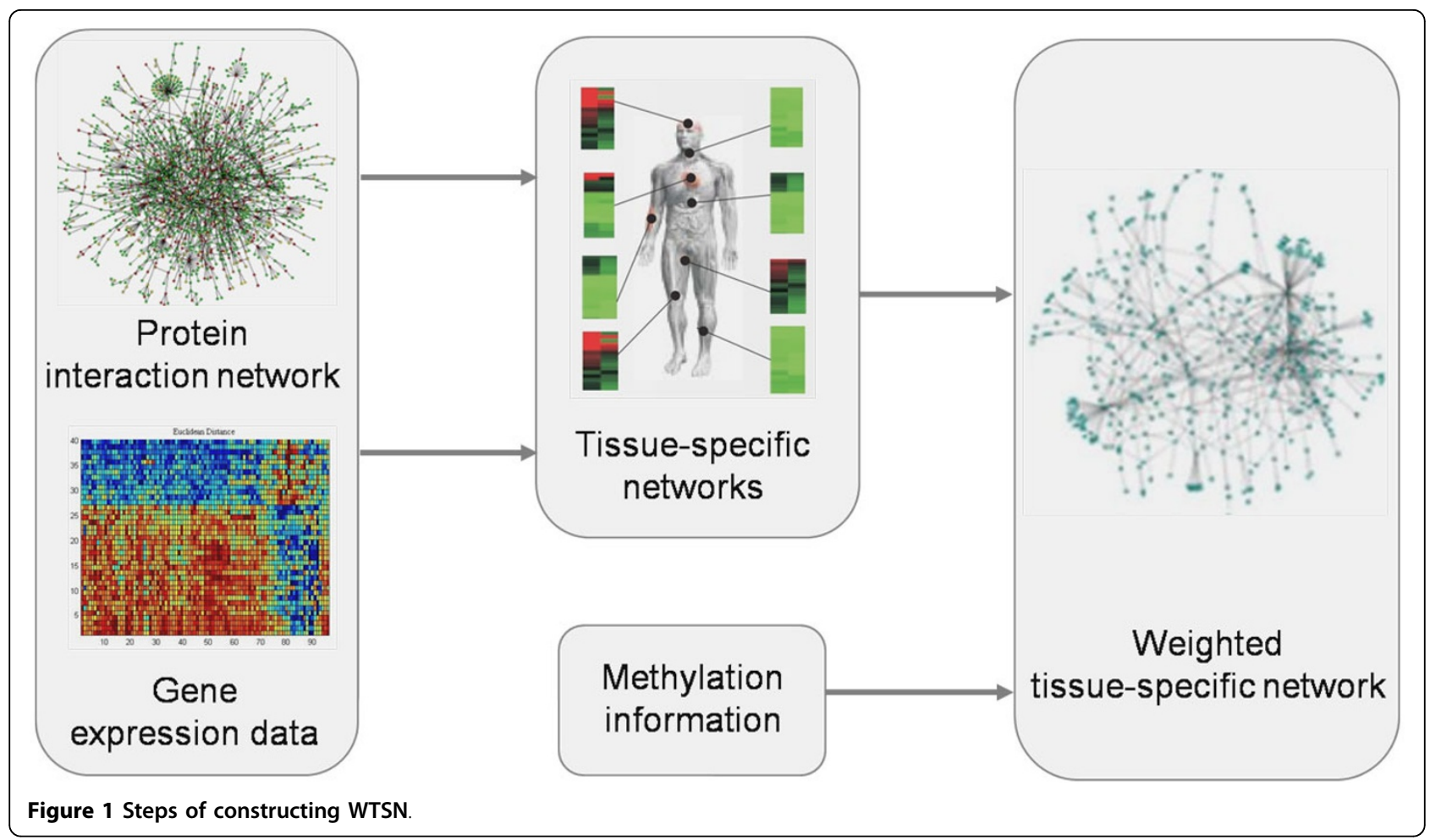

subnetwork is generated by removing the unexpressed nodes and its interactions from the PIN. Then, a final TSN can be constructed by combing the subnetworks for all the disease-related tissues.

In step 3, we further weighted the TSN by using DNA methylation data. For two connected proteins in TSN, the Pearson Correlation Coefficient (PCC) [48-50], as shown in formula (1), was calculated to assess the two proteins' association of the methylation.

$$
\operatorname{PCC}(X, Y)=\frac{\sum x_{i} \cdot y_{i}-\frac{\sum x_{i} \cdot \sum y_{i}}{N}}{\sqrt{\left(\sum x_{i}^{2} \frac{\left(\sum x_{i}\right)^{2}}{N}\right) \cdot\left(\sum y_{i}^{2}-\frac{\left(\sum y_{i}\right)^{2}}{N}\right)}}
$$

where $\mathrm{X}$ and $\mathrm{Y}$ are two proteins which interact with each other in TSN. The variables

$x_{i}$ and $y_{i}$ denote the corresponding DNA methylation value of protein $\mathrm{X}$ and protein $\mathrm{Y}$ at $\mathrm{i}^{\text {th }}$ point, respectively. $\mathrm{N}$ indicates the total number of methylation data for each protein in TSN.

\section{PageRank-based method}

The PageRank algorithm was first proposed by Brin and Page [51], which was used to evaluate webpage and produce an authority value to show the importance of each webpage. The main idea of PageRank is to suppose that a random walker selects chains to be visited according to uniform probability distribution. As the PIN is generally considered as an undirected graph, we implemented PageRank on undirected graph in this paper. It has been shown that the PageRank of an undirected graph is statistically close to the degree distribution. Hence, we compared the results of PageRank-based method and degree-centrality-based method in the section of results. The PageRank algorithm was computed in an iterative way where the probability distribution is used as the input of the next walking of this process.

In this paper, we treated known aberrant methylation genes as seed nodes and set initial quantity value with the use of the seed set, which will enhance the importance of seed nodes in network and solve defects of initial PageRank algorithm. The aberrant methylation data related to specific diseases in PubMeth database [44] were used in this paper.

Before using the PageRank-based method to predict disease-related genes, we perturbed the DNA methylation data 1000 times and recalculated the PCC of the random DNA methylation for the gene pairs in each perturbed methylation dataset. Then, 1000 random WTSNs were obtained and the PageRank algorithm was applied on these 1000 random WTSNs. The average PageRank value for each protein was calculated on the 1000 random WTSNs and it is considered to be distinctiveness if its PageRank value on the WTSN is higher than the average PageRank value on the 1000 random WTSNs. All the proteins in the WTSN were ranked according to their PageRank values in original WTSN and those distinctiveness 
proteins will be outputted as candidate disease-related genes.

\section{Results and discussion}

To validate the effectiveness of the constructed WTSN and the PageRank-based method, we used four different types of PINs: original human PIN, TSN for each special disease, WPIN by using DNA methylation, and WTSN. We applied the PageRank-based method to these four different types of protein interaction networks, and the corresponding results were marked as PR, SPR, WPR, SWPR, respectively. Two important cancers of colon cancer and leukemia were used as case studies here. For each test, the predicted precision was calculated by using the following formula:

$$
\text { precision }=M_{\text {Signature }} / N_{\text {Prediction }}
$$

where $N_{\text {Prediction }}$ is the number of predicted candidate disease-related genes and $M_{\text {Signature }}$ is the number of disease-related genes which can be found in GeneSigDB [52] with the corresponding signatures.

In this paper, the improved PageRank algorithm by using significant analysis is also compared to the degree centrality-based method. In 2011, Liu et al. [32] identified potential disease-related genes by using weighted degree centrality based on the integration of DNA methylation and protein-protein interaction data. Degree centrality (DC) of a given protein in an unweighted network is defined as the number of nodes that directly connect to it. The weighted degree centrality (WDC) of a given protein in a weighted network is defined as the sum of weights of edges connecting the given node and its neighbors. We marked the results of DC as SDC and
SWDC when it being applied on the TSN and the WTSN, respectively.

\section{Identification of disease-related genes from TSN}

To analyze whether the tissue specific information is useful to the identification of disease-related genes, we first applied the PageRank-based method by using significant analysis and degree centrality-based method to the original PIN and the TSN. The experimental results on colon cancer and leukemia were shown in Figure 2. From Figure 2 we can see that both the PageRank method and degree-centrality-based method achieved better results when being applied on TSN than being applied on PIN for colon cancer and leukemia. Especially for colon cancer, the predicted precision of the PageRank algorithm and degree-centrality were improved more than $20 \%$ on average when predicting no more than 100 candidate disease-related genes. For the same cancer, the PageRank-based method by using significant analysis performs a little better than degree centrality-based method when being applied on TSN.

\section{Identification of disease-related genes from WTSN}

In the above subsection, we have shown that tissue specific information contributes to the accurate identification of disease-related genes. In this subsection, we further evaluated the effectiveness of weighting by using DNA methylation and compared the results on WPIN and PIN. As shown in Figure 3 (A), both the PageRankbased method and the degree-centrality-based method achieved better results when being applied on WPIN than on PIN for predicting no more than 80 colon cancer-related candidate genes. Similar results were

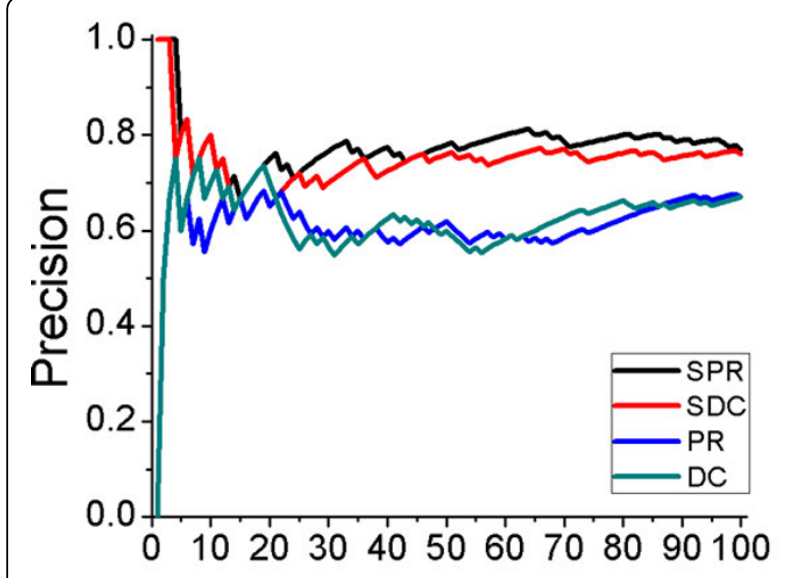

(A)

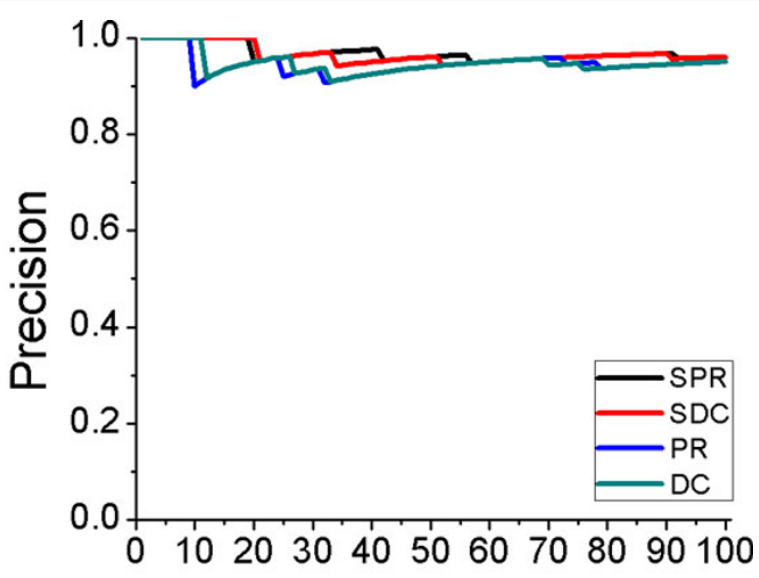

(B)

Figure 2 Comparison of results for PageRank and degree centrality when being applied on the original protein interaction network (PIN) and tissue specific network (TSN). The $x$ axis represents the number of identified disease-related genes. The $y$ axis represents the predicted precision of each result. (A) Results on colon cancer. (B) Results on leukemia. 

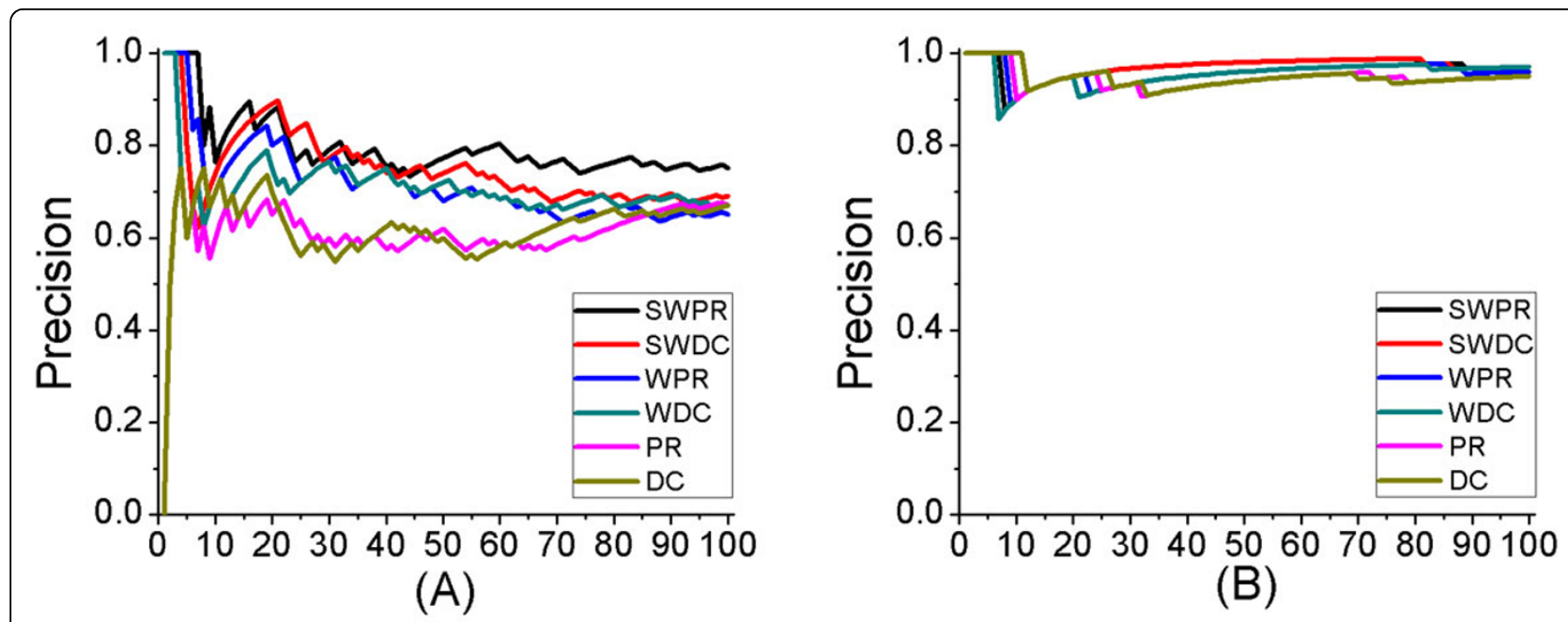

Figure 3 Comparison of results for PageRank and degree centrality when being applied on the original protein interaction network (PIN), weighted protein interaction network (WPIN), and weighted tissue specific network (WTSN). The $x$ axis represents the number of identified disease-related genes. The y axis represents the predicted precision of each result. (A) Results on colon cancer. (B) Results on leukemia.

obtained for leukemia, as shown in Figure 3(B). However, the improvement is not so clear for the high predicted precision on leukemia. The improvement of predicted precision on the WPIN shows that weighting protein interaction network by using DNA methylation contributes to more accurate prediction of diseaserelated genes.

To analyze the contributions of combination of tissuespecific and DNA methylation, we showed the results of PageRank-based method and Degree-centrality-based method both on WPIN and WTSN. From Figure 3 we can see that the predicted precisions of PageRank-based method and Degree-centrality-based method were both further improved when being applied on WTSN. As shown in Figure $3(\mathrm{~A})$, the precision of SWPR is 1.2 times more than that of WPR for predicting no more than 100 colon cancer-related candidates. On the WTSN, the PageRank-based method still performs a little better than the degree centrality-based method. Hence, we further analyzed the candidate disease-related genes predicted by the PageRank-based method. Table 1 and Table 2 showed the top 20 candidate disease-related

Table 1 Top 20 candidate disease-related genes identified by PageRank method with significant analysis from PIN, TSN, WPIN, WTSN, for colon cancer, respectively.

\begin{tabular}{|c|c|c|c|c|c|c|c|}
\hline \multicolumn{2}{|c|}{ WTSN } & \multicolumn{2}{|c|}{ WPIN } & \multicolumn{2}{|c|}{ TSN } & \multicolumn{2}{|c|}{ PIN } \\
\hline Genes & In GeneSigDB & Genes & In GeneSigDB & Genes & In GeneSigDB & Genes & In GeneSigDB \\
\hline $\mathrm{CDH} 1$ & yes & APC & yes & $\mathrm{NR3C1}$ & yes & UBC & no \\
\hline FAS & yes & $\mathrm{CDH} 1$ & yes & MLH1 & yes & ELAVL1 & yes \\
\hline CD44 & yes & FAS & yes & $\mathrm{CDH} 1$ & yes & SUMO2 & yes \\
\hline THBS1 & yes & CD44 & yes & CDKN2A & yes & MYC & yes \\
\hline TIMP3 & yes & THBS1 & yes & ATM & no & GRB2 & no \\
\hline GSTP1 & yes & STK11 & no & FAS & yes & SUMO1 & yes \\
\hline CREBBP & yes & GSTP1 & yes & MGMT & no & SNCA & no \\
\hline STK11 & no & UBC & no & THBS1 & yes & ESR1 & yes \\
\hline HDAC1 & yes & ALX4 & no & CD44 & yes & GABARAPL2 & no \\
\hline UBC & no & HRK & yes & RASSF1 & yes & TP53 & yes \\
\hline $\mathrm{NCL}$ & yes & TIMP3 & yes & STK11 & no & YWHAZ & yes \\
\hline CCND1 & yes & SRC & yes & DAPK1 & yes & GABARAPL1 & yes \\
\hline PRKDC & yes & $\mathrm{NCL}$ & yes & CHFR & no & GABARAP & no \\
\hline SFN & yes & CREBBP & yes & GSTP1 & yes & TRAF6 & yes \\
\hline ELAVL1 & yes & HDAC1 & yes & UBC & no & RAD23A & yes \\
\hline
\end{tabular}


Table 1 Top 20 candidate disease-related genes identified by PageRank method with significant analysis from PIN, TSN, WPIN, WTSN, for colon cancer, respectively. (Continued)

\begin{tabular}{cccccccc}
\hline TGM2 & yes & SFRP1 & yes & TIMP3 & yes & EP300 & no \\
\hline FYN & no & ELAVL1 & yes & HSP90AA1 & yes & EGFR & yes \\
\hline FN1 & yes & CCND1 & yes & TP53 & yes & SRC & yes \\
\hline ACTR3 & yes & PRKDC & yes & SFRP1 & yes & YWHAG & yes \\
\hline MMP14 & yes & FYN & no & PML & yes & ESR2 & no \\
\hline
\end{tabular}

Table 2 Top 20 candidate disease-related genes identified by PageRank method with significant analysis from PIN, TSN, WPIN, WTSN, for Leukemia, respectively.

\begin{tabular}{|c|c|c|c|c|c|c|c|}
\hline \multicolumn{2}{|c|}{ WTSN } & \multicolumn{2}{|c|}{ WPIN } & \multicolumn{2}{|c|}{ TSN } & \multicolumn{2}{|c|}{ PIN } \\
\hline Genes & In GeneSigDB & Genes & In GeneSigDB & Genes & In GeneSigDB & Genes & In GeneSigDB \\
\hline ABL1 & yes & ESR1 & yes & ESR1 & yes & ESR1 & yes \\
\hline CDKN1A & yes & ABL1 & yes & ABL1 & yes & ABL1 & yes \\
\hline MLH1 & yes & CDKN1A & yes & MLH1 & yes & RB1 & yes \\
\hline MGMT & yes & CCND1 & yes & RB1 & yes & CDKN1A & yes \\
\hline LMNA & yes & CDKN2A & yes & CDKN1A & yes & RARA & yes \\
\hline NROB2 & yes & MLH1 & yes & CDKN2A & yes & MLH1 & yes \\
\hline CHFR & yes & PARK2 & no & PTPN6 & yes & $\mathrm{CDH} 1$ & yes \\
\hline DIABLO & yes & MME & yes & SYK & yes & PTPN6 & yes \\
\hline CEBPD & yes & MYOD1 & yes & PTEN & yes & CDKN2A & yes \\
\hline DAPK1 & yes & LMNA & yes & MGMT & yes & PARK2 & no \\
\hline GRB2 & yes & NROB2 & yes & $\mathrm{HCK}$ & yes & SYK & yes \\
\hline ACTB & yes & MGMT & yes & THBS1 & yes & CCND1 & yes \\
\hline $\mathrm{HDAC1}$ & yes & APAF1 & yes & LMNA & yes & TP73 & yes \\
\hline HSP90AA1 & yes & PGR & yes & NROB2 & yes & MYOD1 & yes \\
\hline PAX6 & yes & AHR & yes & UBC & yes & PTEN & yes \\
\hline FYN & yes & DAPK1 & yes & CHFR & yes & LMNA & yes \\
\hline EEF1A1 & yes & PAX6 & yes & DAPK1 & yes & THBS1 & yes \\
\hline JAK1 & yes & CIITA & yes & GSTP1 & yes & MGMT & yes \\
\hline CRKL & yes & CHFR & yes & RARB & yes & $\mathrm{HCK}$ & yes \\
\hline CCNB1 & yes & $\mathrm{HIC1}$ & yes & DIABLO & no & NROB2 & yes \\
\hline
\end{tabular}

genes of colon cancer and Leukemia, respectively, which were identified by the PageRank method with significant analysis from PIN, TSN, WPIN, WTSN.

As shown in Table 1, out of the top 20 candidate disease-related genes of colon cancer, 17 genes were found to have gene signatures in GeneSigDB. The number of true colon cancer-related genes identified from WTSN is higher than that identified from PIN, WPIN and TSN. For the unknown gene STK11 identified from WTSN, we found that it has been reported to contribute to the development of both sporadic and familial forms of cancer and germline and somatic genetic alterations of the STK11/LKB1 gene may play a causal role in carcinogenesis [53]. For leukemia, we were delighted to see that all the top 20 candidate disease-related genes were included in GeneSigDB. The top 100 candidate disease-related genes of colon cancer and Leukemia can be seen in additional file 1 and additional file 2 respectively.

\section{Conclusion}

The primary purpose of this study is to use WTSN to predict disease-related genes. We proposed a PageRank-based method to identify disease-related genes from WTSN. Firstly, TSN was constructed by combining PIN and gene expression data. Secondly, WTSN was constructed by weighting TSN with methylation information. Finally, an improved PageRank algorithm was used to predict disease-related genes by using significance analysis based on WTSN. To validate the effectiveness of the proposed method, we constructed PIN, WPIN, TSN, WTSN for colon cancer and leukemia, respectively. The experimental results on colon cancer and leukemia show that the 
combination of tissue-specific data and DNA methylation can help to identify disease-related genes more accurately. Moreover, the PageRank-based method was effective to predict disease-related genes on the case studies of colon cancer and leukemia.

\section{Additional material}

Additional File 1: The top 100 candidate disease-related genes of colon cancer

Additional File 2: The top 100 candidate disease-related genes of Leukemia

\section{Competing interests}

The authors declare that they have no competing interests.

\section{Authors' contributions}

JYZ and QL obtained the protein-protein interaction data, tissue-specific data, methylation information and GeneSigDB. ML, JYZ and QL designed the method to predict disease-related genes. JYZ and ML analyzed the results. $\mathrm{ML}, \mathrm{JXW}$ and FXW drafted the manuscript together. All authors have read and approved the manuscript.

\section{Acknowledgements}

This work is supported in part by the National Natural Science Foundation of China under Grant No. 61370024, No.61379108, No.61232001, and the Program for New Century Excellent Talents in University (NCET-12-0547).

\section{Declarations}

The publication costs for this article were funded by the National Natural Science Foundation of China under Grant No. 61370024

This article has been published as part of BMC Medical Genomics Volume 7 Supplement 2, 2014: IEEE International Conference on Bioinformatics and Biomedicine (BIBM 2013): Bioinformatics in Medical Genomics. The full contents of the supplement are available online at http://www. biomedcentral.com/bmcmedgenomics/supplements/7/S2.

\section{Authors' details}

'School of Information Science and Engineering, Central South University, Changsha 410083, Hunan, P. R. China. ${ }^{2}$ College of Engineering, University of Saskatchewan, 57 Campus Dr., Saskatoon, SK Canada.

\section{Published: 22 October 2014}

\section{References}

1. Wang J, Li M, Deng Y, Pan Y: Recent advances in clustering methods for protein interaction networks. BMC Genomics 2010, 11(Suppl 3):S10.

2. Li M, Wu X, Wang J, Pan Y: Towards the identification of protein complexes and functional modules by integrating PPI network and gene expression data. BMC Bioinformatics 2012, 13(1):109.

3. Zhao B, Wang J, Li M, Wu F-X, Pan Yi: Detecting Protein Complexes Based on Uncertain Graph Model. IEEE/ACM Transactions on Computational Biology and Bioinformatics 2014, 11(3):486-497.

4. Keong $\mathrm{H}$, Mason $\mathrm{SP}$, Barabai $\mathrm{AL}$, et al: Lethality and centrality in protein networks. Nature 2001, 411(6833):41-42.

5. Zhong J, Wang J, Peng W, et al: Prediction of essential proteins based on gene expression programming. BMC Genomics 2013, 14(4):18.

6. Wang J, Peng W, Wu F X: Computational approaches to predicting essential proteins: a survey. PROTEOMICS-Clinical Applications 2013, 7(1-2):181-192.

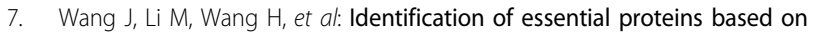
edge clustering coefficient. Computational Biology and Bioinformatics 2012, 9(4):1070-1080, IEEE/ACM Transactions on.

8. Saket Navlakha, Carl Kingsford: The power of protein interaction networks for associating genes with diseases. Bioinformatics 2010, 26(8):1057-1063.
9. Lage $K$, et al: A human phenome-interactome network of protein complexes implicated in genetic disorders. Nature Biotechnol 2007, 25(23):309-316.

10. Wu $X$, et al: Network-based global inference of human disease genes Molecular Systems Biology 2008, 4(1):189.

11. Linghu B, et al: Genome-wide prioritization of disease genes and identification of disease-disease associations from an integrated human functional linkage network. Genome Biology 2009, 10:R91.

12. MOti, Snel B, Huynen MA, Brunner HG: Predicting disease genes using protein-protein interactions interactions. Journal of medical genetics 2006, 43(8):691-698.

13. Gozde Kar, Attila Gursoy, Ozlem Keskin: Human Cancer Protein-Protein Interaction Network: A Structural Perspective. PLOS Computational Biology 2009, 5(12):1-18

14. Sreenivas Chavali, Fredrik Barrenas, Kartiek Kanduri, et al: Network properties of human disease genes with pleiotropic effects. BMC Systems Biology 2010, 4(1):78.

15. Jingchun Sun, Peilin Jia, Ayman HFanous, et al: Schizophrenia Gene Networks and Pathways and Their Applications for Novel Candidate Gene Selection. PLOS ONE 2010, 5(6):e11351.

16. Xianzhen $\mathrm{Xu}$, Yongjin Li: Discovering disease-genes by topological features in human protein-protein interaction network. Bioinformatics 2006, 22(22):2800-2805.

17. Sun PG, Gao L, Han S: Prediction of human disease-related gene clusters by clustering analysis. International journal of biological sciences 2011, 7(1):61.

18. Shrivastava N: Graph summarization with bounded error. Proceedings of the 2008 ACM SIGMOD international conference on Management of data 2008, 419-432.

19. Stijn Van Dongen: Graph clustering via a discrete uncoupling process. SIAM Journal on Matrix Analysis and Applications 2008, 30(1):121-141.

20. Saket Navlakha, Niranjan Nagarajan, James White, Navlakha S, Rastogi R, et al: Finding Biologically Accurate Clusterings in Hierarchical Tree Decompositions Using the Variation of Information. Journal of Computational Biology 2010, 17(3):503-516.

21. Li M, Chen J, Wang J, et al: Modifying the DPClus algorithm for identifying protein complexes based on new topological structures. BMC bioinformatics 2008, 9(1):398.

22. Ding $X$, Wang $W$, Peng $X$, Wang J: Mining protein complexes from PPI networks using the minimum vertex cut. Tsinghua Science and Technology 2012, 17(6):674-681.

23. Wang J, Li M, Chen J, et al: A fast hierarchical clustering algorithm for functional modules discovery in protein interaction networks. IEEE/ACM Trans Comput Biol Bioinform 2011, 8(3):607-620.

24. Erten Sinen, Mehmet Koyutürk: Role of Centrality in Network-Based Prioritization of Disease Genes[M]//Evolutionary Computation, Machine Learning and Data Mining in Bioinformatics Lecture Notes in Computer Science. Evolutionary Computation, Machine Learning and Data Mining in Bioinformatics. Lecture Notes in Computer Science 2010, 6023:13-25.

25. Montanez G, Cho Y-R: Predicting False Positives of Protein-Protein Interaction Data by Semantic Similarity Measures. Current Bioinformatics 2013, 8(3):339-346.

26. Li M, Zheng $R$, Zhang $H$, Wang J, Pan Y: Effective identification of essential proteins based on priori knowledge, network topology and gene expressions. Methods 2014, 67(3):325-333.

27. Wang J, Peng $X$, Peng $W$, et al: Dynamic protein interaction network construction and applications. Proteomics 2014, 8(4-5):338-352.

28. Wang J, Peng $X$, Li M, et al: Construction and application of dynamic protein interaction network based on time course gene expression data. Proteomics 2013, 13(2):301-312.

29. Tang $X$, Feng $Q$, Wang J, et al: Clustering based on multiple biological information: approach for predicting protein complexes. IET systems biology 2013, 7(5):223-230.

30. Peng W, Wang J, Wang W, Liu Q, Wu FX, Pan Y: Iteration method for predicting essential proteins based on orthology and protein-protein interaction networks. BMC systems biology 2012, 6(1):87.

31. Mahmoud AMahdavi, Yen-Han Lin: False positive reduction in proteinprotein interaction predictions using gene ontology annotations. BMC Bioinformatics 2007, 8:262.

32. Liu H, Su J, Li J, et al: Prioritizing cancer-related genes with aberrant methylation based on a weighted protein-protein interaction network. BMC systems biology 2011, 5(1):158 
33. Shlomi T, et al: Network-based prediction of human tissue-specific metabolism. Nature Biotechnol 2008, 26(9):1003-1010.

34. Waldman YY, Tuller T, Shlomi T, et al: Translation efficiency in humans: tissue specificity global optimization and differences between developmental stages. Nucleic Acids Research 2010, 38(9):2964-2974.

35. Bossi A, Lehner B: Tissue specificity and the human protein interaction network. Molecular Systems Biology 2009, 5(1):260.

36. Magger $\mathrm{O}$, Waldman $\mathrm{YY}$, Ruppin E, et al: Enhancing the prioritization of disease-causing genes through tissue specific protein interaction networks. PLoS Computational Biology 2012, 8(9):e1002690.

37. Xenarios I, Rice DW, Salwinski L, et al: DIP: the database of interacting proteins. Nucleic Acids Res 2000, 28(1):289-291.

38. Kerrien S, Alam-Faruque Y, Aranda B, Bancarz I, Bridge A, Derow C, Dimmer E, Feuermann M, Friedrichsen A, Huntley R, et al: IntAct-open source resource for molecular interaction data. Nucleic acids research 2007, 35(suppl 1):D561-D565.

39. Ceol A, Chatr Aryamontri A, Licata L, Peluso D, Briganti L, Perfetto L, Castagnoli L, Cesareni G: MINT, the molecular interaction database: 2009 update. Nucleic Acids Res 2010, 38(suppl 1):D532-539.

40. Stark C, Breitkreutz BJ, Reguly T, et al: BioGRID: a general repository for interaction datasets. Nucleic acids research 2006, 34(suppl 1):D535-D539.

41. Keshava Prasad TS, Goel R, Kandasamy K, Keerthikumar S, Kumar S, Mathivanan S, Telikicherla D, Raju R, Shafreen B, Venugopal A, et al: Human Protein Reference Database-2009 update. Nucleic acids research 2009, 37(suppl 1):D767-D772.

42. Su Al, Wiltshire T, Batalov $S$, et al: A gene atlas of the mouse and human protein-encoding transcriptomes. Proceedings of the National Academy Sciences of the United States of America 2004, 101(16):6062-6067.

43. Chen Yao, Hongdong Li, Xiaopei Shen, Zheng He, Lang He, Zheng Guo: Reproducibility and Concordance of Differential DNA Methylation and Gene Expression in Cancer. PLOS ONE 2012, 7(1):e29686.

44. Ongenaert M, Van Neste L, De Meyer T, et al: PubMeth: a cancer methylation database combining text-mining and expert annotation. Nucleic Acids Research 2008, 36(suppl 1):D842-D846.

45. Culhane AC, Schwarzl T, Sultana R, et al: GeneSigDB-a curated database of gene expression signatures. Nucleic Acids Res. 2012, 40(D):D1060-D1066.

46. Lage $K$, Hansen NT, Karlberg EO, et al: A large-scale analysis of tissuespecific pathology and gene expression of human disease genes and complexes. Proceedings of the National Academy of Sciences 2008, 105(52):20870-20875

47. Lopes TJ, Schaefer M, Shoemaker J, et al: Tissue-specific subnetworks and characteristics of publicly available human protein interaction databases. Bioinformatics 2011, 27(17):2414-2421.

48. Tang $X$, Wang J, Zhong J, Pan Y: Predicting Essential proteins based on Weighted Degree Centrality. Computational Biology and Bioinformatics, IEEE/ACM Transactions 2014, 11(2):407-418.

49. Li M, Zhang H, Wang J, Pan Y: A new essential protein discovery method based on the integration of protein-protein interaction and gene expression data. BMC systems biology 2012, 6(1):15.

50. Eggers JJ, Bauml R, Tzschoppe R, et al: Scalar costa scheme for information embedding. Signal Processing, IEEE Transactions on 2003, 51(4):1003-1019.

51. Brin S, Page L: The anatomy of a large-scale hypertextual Web search engine. Computer networks and ISDN systems 1998, 30(1):107-117 [http:// infolab.stanford.edu/ backrub/google.html].

52. Culhane AC, Schröder MS, Sultana R, et al: GeneSigDB: a manually curated database and resource for analysis of gene expression signatures. Nucleic acids research 2012, 40(D1):1060-1066.

53. Su GH, Hruban RH, Bansal RK, et al: Germline and Somatic Mutations of the STK11/LKB1 Peutz-Jeghers Gene in Pancreatic and Biliary Cancers. The American Journal of Pathology 1999, 154(6):1835-1840.

doi:10.1186/1755-8794-7-S2-S4

Cite this article as: Li et al:: Prediction of disease-related genes based on weighted tissue-specific networks by using DNA methylation. BMC Medical Genomics 2014 7(Suppl 2):S4. 